\title{
The Causal Role of Fatigue in the Stress-Perceived Health Relationship: A MetroNet Study
}

\author{
Sham Maghout-Juratli, MD, MPH, James Janisse, PhD, Kendra Schwartz, MD, MSPH, \\ and Bengt B. Arnetz, MD, PhD
}

Background: We conducted a cross-sectional survey of 4 primary care MetroNet centers in metropolitan Detroit. Our objective was to describe the causal role of fatigue in the relationship among stress, stress resiliency, and perceived health in primary care. Fatigue is a public health problem that has been linked to stress and poor health. The causal role of fatigue between stress and perceived health is unknown.

Methods: Four hundred surveys were distributed to adult patients in 4 primary care centers in metropolitan Detroit between 2006 and 2007. Internal consistency reliabilities and principal factor analyses were calculated for the key psychological scales. Perceived health is the primary outcome. Path models were used to study the relationship among stress, fatigue, and perceived health. We also modeled the impact of select stress resiliency factors including sleep, recovery, and social support.

Results: Of the 400 distributed surveys, 315 (78.7\%) had a response rate of $70 \%$ or more and were included in the analysis. Respondents were predominantly middle aged (median age, 43 years); female (58.7\%); and African American (52.0\%). The majority worked full time (56.5\%); did not have a college degree $(77.7 \%)$; and were not married $(55.2 \%)$. Fatigue was reported by $59 \%$ of respondents, $42.7 \%$ of which was unexplained. The path model supported the causal role of fatigue between stress and perceived health. The positive effects of sleep, recovery, and social support on fatigue, stress, and perceived health were validated.

Conclusion: Fatigue was common in this metropolitan primary care environment and completely mediated the relationship between stress and poor perceived health. Therefore, stress, when significant enough to cause fatigue, may lead to poor health. (J Am Board Fam Med 2010;23:212-219.)

Keywords: Fatigue, Stress, Perceived Health

Fatigue is a common and costly public health problem with well-recognized health, safety, and performance implications. $^{1-3}$ It affects up to $40 \%$ of the population and is a frequent complaint by primary care patients. ${ }^{4,5}$ The subjective nature of fatigue coupled with the frequent lack of medical explanations renders it a true clinical challenge for primary care providers. ${ }^{4}$ As with other medically unexplained symptoms, ${ }^{6}$ stress is frequently impli-

This article was externally peer reviewed.

Submitted 1 June 2009; revised 23 July 2009; accepted 28 July 2009.

From the Occupational and Environmental Health Division (SM-J, BBA), the Division of Practice-Based Research (KS), the Department of Family Medicine and Public Health Sciences (SM-J, BBA, JJ, KS), Wayne State University, Detroit, Michigan.

Funding: Funding from Wayne State University School of Medicine research funds and Skandia Insurance.

Conflict of interest: none declared.

Corresponding author: Sham Maghout-Juratli, MD, Wayne State University, 3800 Woodward Avenue, Suite 808, Detroit, MI 48201 (E-mail: smjuratli@med.wayne.edu). cated in medically unexplained fatigue and in diseases hallmarked by fatigue, such as fibromyalgia and chronic fatigue syndrome. ${ }^{7-9}$ In addition, essential components of stress resiliency, such as social support, sleep, and recovery, are inversely related to fatigue. ${ }^{10}$ The detrimental effect of stress on health has been established, as have the stress implications of poor health. ${ }^{11-15}$ Therefore, fatigue is an integral part of the intertwined stress-health relationship. However, the causal role of fatigue in this relationship is unknown.

We tested the causal role of fatigue in the relationship between stress and perceived health in a cross-sectional sample of attendants to 4 primary care centers in metropolitan Detroit. This sample allowed us to model stress and fatigue in different primary care settings where both problems are common. We focused not only on stressors but, importantly, also on possible stress resiliency factors, including sleep, recovery, and social support. This offered us the op- 
portunity to model a more ecologically relevant data set.

Perceived health was our primary outcome because it is a validated predictor of future morbidity and mortality ${ }^{16-19}$ and a useful and feasible clinical tool. ${ }^{20,21}$ This study will help advance the understanding of the complex relationship between stress, fatigue, and perceived health in primary care.

\section{Methods}

\section{Procedure}

After obtaining the appropriate institutional ethical approval from Wayne State University, 400 selfadministered, anonymous questionnaires were distributed by a designated front desk person or a research assistant at 4 participating primary care centers between November 2006 and December 2007. These centers were all part of MetroNet, a practice-based research network in metropolitan Detroit. ${ }^{22}$ MetroNet is a consortium of 24 primary care practices and was established in 2001 to advance practice-based research focused primarily on underserved patient populations. The 4 MetroNet practices were selected because of the racial and socioeconomic diversity they represented. In each of the 4 centers, 100 surveys were offered to 100 consecutive patients. Each respondent was informed of the purpose of the survey with an introductory information sheet. If a patient chose not to participate, his or her survey was turned in blank and not offered to another patient. Returned surveys were collected in designated boxes.

\section{Survey Instrument}

The survey consisted of 63 questions about select sociodemographic characteristics, and visual analog scale (VAS)-type items $(0-100)$ that measured fatigue, stress, perceived health, sleep, recovery, and general symptoms. The VAS items were anchored with opposing terms such as "never" and "daily" or "not at all" and "very much so." The stress and fatigue items have been previously validated in various combinations. ${ }^{23-25}$ Internal consistency was assessed with Cronbach $\alpha$ coefficient (all $\alpha$ coefficients were $\geq 0.80$ ) and dimensionality was assessed with confirmatory factor analysis using the principal components method and varimax rotation (all loaded on one factor that explained $60 \%$ or more of the variance). A score pertinent to each of these constructs was generated by averaging the scores on the corresponding VAS items. Higher fatigue, stress, and general symptoms scores indicated more fatigue, stress, and general symptoms, respectively. Higher perceived health, recovery, and sleep scores indicated better perceived health, recovery, and sleep, respectively.

Fatigue was measured with 6 VAS items that reflected physical and mental fatigue. Two items measured physical fatigue: level of energy and exhaustion; 4 items measured mental fatigue ${ }^{26}$ : moodiness, restlessness, irritation, and difficulty concentrating. These items were adapted from the Brief Fatigue Syndrome Scale and the mental energy subscale of the Quality Work Competence questionnaire, which are validated and have been used in a number of studies, including prospective, controlled intervention studies targeting mental energy as well as fatigue. ${ }^{24,25}$ In agreement with previously suggested cutoff levels, ${ }^{24}$ a fatigue score of $\geq 40$ on a $100 \%$ scale indicated fatigue and a score of $\geq 75$ indicated severe fatigue.

Perceived stress was measured with 4 VAS items that assessed the extent of present and past pressure and stress. Consistent with the suggested cutoff points for the fatigue scale, a score of $\geq 40$ was indicative of stress and a score of $\geq 75$ was indicative of severe stress.

Recovery was measured with 4 VAS items that assessed the ability to rest, relax, recharge, and mobilize extra strength when needed. Sleep quality was measured with 5 VAS items that assessed perceived sleep quality presently and during the past year, restorative sleep, and difficulty initiating or sustaining sleep. Social support was assessed with a single VAS item concerned with the perception of social support. Social support, sleep, and recovery were the selected stress resiliency factors.

Perceived health was measured with 2 VAS items concerned with general health perceptions presently and during the past year. ${ }^{19-21}$ General symptoms were assessed with 8 VAS items measuring the frequency of headache, anxiety, dizziness, pain, altered appetite, gastrointestinal symptoms, and cardiac symptoms. Health status was determined by the number of diagnosed diseases (diabetes, hypertension, cancer, prolonged pain, asthma, allergies, and cardiovascular and mental disorders) and was categorized as none, 1 to 2 , and $>2$ current diseases. 
For the purposes of the analysis, race was categorized as African American, white, and other. In the path model only whites and African Americans were included because of the small number and heterogeneity of the "other" race group. Exercise was categorized as "mostly idle," "some regular exercise," and "regular moderate exercise 2 to 3 times a week. " Body size was based on self-reported height and weight, which was converted to body mass index (BMI) and defined as normal (BMI, 18.5-24.9 kg/m²); overweight (BMI, 25.0$29.9 \mathrm{~kg} / \mathrm{m}^{2}$ ); obese (BMI, 30.0-39.9 $\mathrm{kg} / \mathrm{m}^{2}$ ); and morbidly obese (BMI, $\geq 40 \mathrm{~kg} / \mathrm{m}^{2}$ ).

\section{Statistical Analysis}

Multiple imputation methods using SPSS Multiple Imputation (version 17.0, SPSS, Inx., Chicago, IL) were used to manage the nonresponse items. Summary statistics for the main scales (stress, fatigue, sleep, and recovery) were compared using complete case analysis and multiple imputation analysis and revealed very close values (data not presented). Manifest path analysis using LISREL version 8.72 (Scientific Software International, Inc., Lincolnwood, IL) was performed to test the hypothesized causal role of fatigue in the relationship between stress and perceived health.

Model fit was assessed using the comparative fit index (CFI), root mean square error of approximation (RMSEA), and 90\% CI for RMSEA. CFI $>0.95$ and RMSEA $<0.06$ indicated a good-fitting model. ${ }^{27}$ Paths that were not significant at $P \leq .05$ were removed from the model. Modifications to the model were based on both substantive clinical and statistical grounds. All $P$ values were based on 2 -sided tests of statistical significance, which was set at 0.05 .

\section{Results}

\section{Study Population}

Of the 400 distributed surveys, 65 were either unreturned or returned blank $(16.2 \%)$ and 20 were missing $>30 \%$ of the responses $(5 \%)$ and were excluded from the analysis. Three hundred and fifteen patients answered $>70 \%$ of the questions and were included in the analysis $(\mathrm{n}=315 ; 78.7 \%)$. Of the $315,219(65.4 \%)$ had complete data and only $5.7 \%$ of the data were missing.

Table 1 summarizes the characteristics of the 315 respondents. Respondents tended to be mid-
Table 1. Characteristics of Primary Care Patients in Metropolitan Detroit $(n=315)$

\begin{tabular}{lr}
\hline Characteristic & $\mathrm{n}(\%)$ \\
\hline Female & $185(58.7)$ \\
Age, years (mean, SD) & $44.6(14.9)$ \\
Race & \\
White & $127(40.3)$ \\
Black & $164(52.0)$ \\
Other & $24(7.6)$ \\
Education & \\
$\leq$ High school & $104(33.0)$ \\
Some college & $133(42.2)$ \\
$\geq$ College degree & $70(22.2)$ \\
Marital status & \\
Cohabitating & $141(44.7)$ \\
Single & $106(33.6)$ \\
Widowed/divorced & $68(21.5)$ \\
Employment status & \\
Unemployed & $98(31.1)$ \\
Part time & $38(12.0)$ \\
Full time & $178(56.5)$ \\
Body mass index & $213(67.6)$ \\
Normal & \\
Overweight & $137(43.4)$ \\
Obese & $96(30.4)$ \\
Morbidly obese & $95(30.1)$ \\
Exercise & \\
None & \\
Some regular exercise & \\
Regular moderate exercise & \\
Current smokers & \\
Diseases present & $36(23.8)$ \\
0 & \\
1-2 & \\
\hline & \\
\hline &
\end{tabular}

*A fatigue case is defined by a fatigue score of $\geq 40$.

${ }^{\dagger} \mathrm{A}$ stress case is defined by a perceived stress score of $\geq 40$.

dle-aged (median age, 43 years; mean age, 44.6 years). More than half were women ( $\mathrm{n}=185$; $58.7 \%$ ) and the majority did not have college degree $(n=245 ; 77.7 \%)$. Close to half were either married or living with a significant other $(n=141$; $44.7 \%)$. A little more than half identified themselves as African American $(\mathrm{n}=164 ; 52.0 \%)$ and $40 \%$ identified as white $(\mathrm{n}=127 ; 40.3 \%)$. The remaining patients were Native American $(n=4)$, Asian $(\mathrm{n}=5)$, and other $(\mathrm{n}=15)$.

The majority were working, either full time ( $\mathrm{n}=$ $178 ; 56.5 \%)$ or part time $(\mathrm{n}=38 ; 12.0 \%)$. Part- 
Figure 1. A path model analysis showing the relationship between stress, fatigue, and perceived health. The standardized coefficients are reported on the corresponding arrows. ${ }^{\mathrm{a} P}<.001 ;{ }^{\mathrm{b}} \boldsymbol{P}<.01$; ${ }^{\mathrm{C} P}<.05$

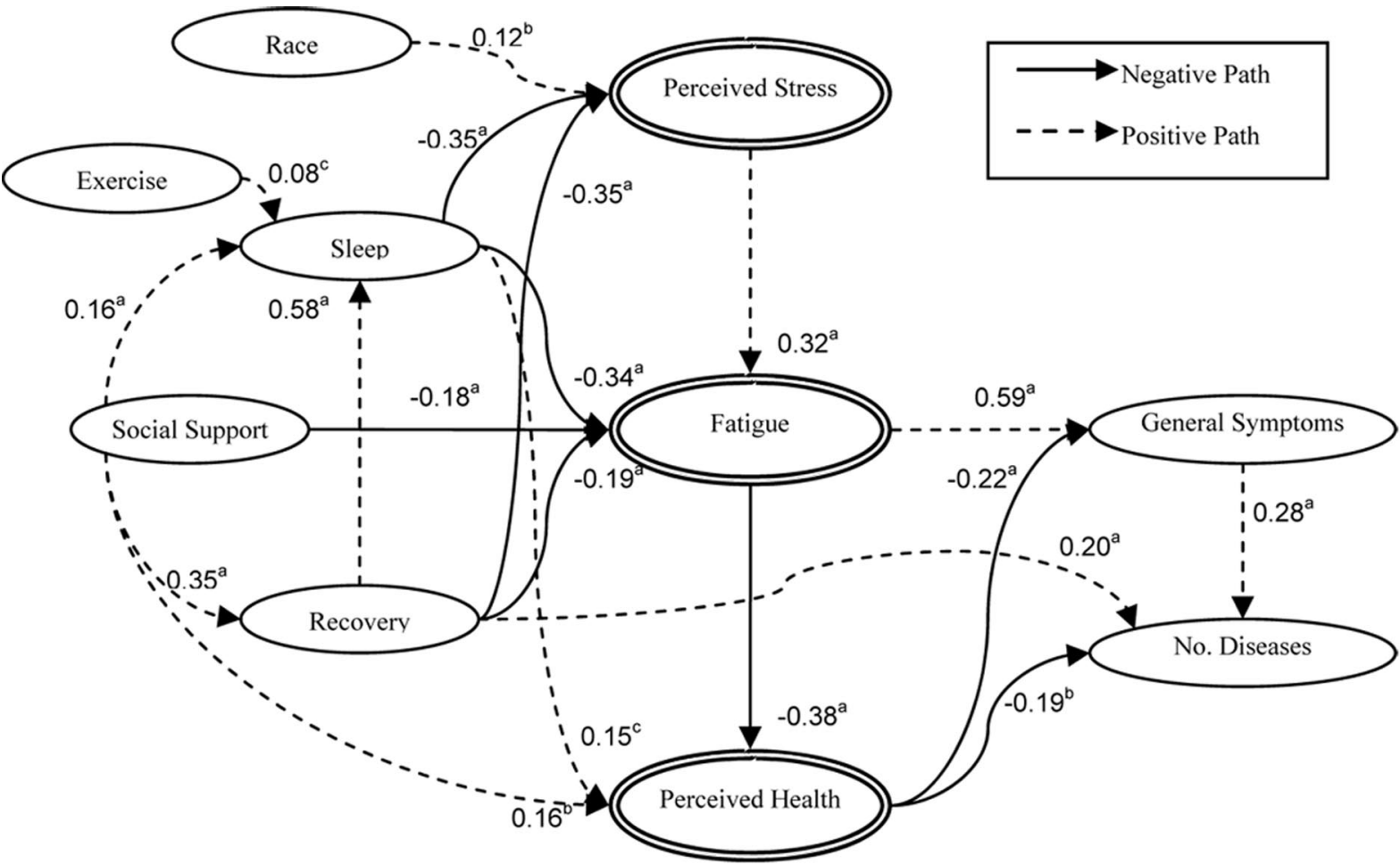

time workers' views of their work were evenly split between positive $(\mathrm{n}=16 ; 41.0 \%)$ and negative $(\mathrm{n}=$ $17 ; 43.5 \%)$ whereas full-time workers held predominantly positive views $(\mathrm{n}=108 ; 60.6 \%)$.

The vast majority of respondents were overweight ( $\mathrm{n}=240 ; 76.1 \%)$, and $11 \%$ were morbidly obese ( $\mathrm{n}=36 ; 11.4 \%)$. Approximately a third were current smokers $(\mathrm{n}=95 ; 30.1 \%)$. Thirty percent of respondents reported regular moderate exercise at least twice a week $(\mathrm{n}=96 ; 30.4 \%)$.

Fifty-three percent reported one or more present diagnosed diseases $(\mathrm{n}=167 ; 53.0 \%)$, with hypertension being the most common ( $\mathrm{n}=74$; $23.4 \%$ ), followed by prolonged pain ( $\mathrm{n}=55$; $17.4 \%)$, allergic disorders ( $\mathrm{n}=49 ; 15.5 \%)$, and mental disorders $(\mathrm{n}=43 ; 13.6 \%)$. Twenty-seven percent had 2 or more medical problems $(\mathrm{n}=86$; $27.3 \%$ ).

Stress and fatigue were common. Fatigue was present in $59.0 \%$ of respondents $(\mathrm{n}=186)$ and severe fatigue was present in $17 \%(n=55,17.4 \%)$. Forty-two percent of those fatigued had no medical or psychiatric illness $(\mathrm{n}=79 ; 42.7 \%)$. Stress was present in $67.6 \%$ of respondents. Severe stress was present in $30 \%$ of respondents ( $\mathrm{n}=94 ; 29.8 \%)$.

\section{Path Analysis}

Figure 1 presents the final path model. The arrows indicate the direct effects between variables and the regression coefficients indicate the magnitude and the strength of these effects. The model fit the data well $\left(\chi^{2}=37.63[23, \mathrm{n}=291] ; P=.028\right.$; RMSEA, 0.045; 90\% CI, 0.01-0.07; CFI, 0.99).

\section{Direct Effects}

Perceived health was predicted by better sleep $(P=$ $.03)$, less fatigue $(P \leq .001)$, and better social support $(P=.003)$. Sleep, recovery, and race had direct effects on perceived stress. White respondents had higher levels of perceived stress than their AfricanAmerican counterparts $(p=.007)$. Better sleep $(P \leq .001)$ and recovery $(P \leq .001)$ predicted lower perceived stress.

\section{Indirect Effects}

Perceived stress had significant indirect effects on perceived health $(P \leq .001)$, general symptoms $(P \leq .001)$, and number of diseases $(P \leq .001)$. Increased levels of perceived stress predicted lower perceived health and increased general symptoms and number of diseases. 
Recovery had an indirect effect on the number of diseases $(P \leq .001)$ that was in the opposite direction of the direct effect $(P=.001)$. The total standardized effect of recovery on the number of diseases was insignificant $(P=.61)$.

\section{Discussion}

This cross-sectional study of primary care patients supported the causal role of fatigue in the relationship between stress and perceived health. When stress is associated with fatigue, stress is associated with poor perceived health; when stress is not associated with fatigue, the association between stress and perceived health is not significant. The positive effects of sleep, recovery, and social support on stress, fatigue, and perceived health were validated.

We found a high prevalence of fatigue in our sample; $59.0 \%$ reported fatigue and $17.4 \%$ reported severe fatigue of at least 1 month's duration. Consistent with the relevant literature about fatigue, medical and psychiatric explanations were often lacking ${ }^{4,28} ; 42.7 \%$ of those fatigued had no medical or psychiatric explanation for their symptoms. These rates are higher than rates reported previously, which ranged between $2 \%$ and $38 \%$ depending on the case definition and the population studied. ${ }^{1,4,5,29-31}$ Moreover, the prevalence of fatigue is sensitive to the chosen scale and threshold. ${ }^{32}$ Nonetheless, unexplained prolonged fatigue is a common problem among this population. We used validated questions that reflected essential mental and physical fatigue experiences and had been proven sensitive in ascertaining improvement in fatigue in controlled prospective studies of antifatigue interventions targeting cognition, exercise, sleep, and nutrition. ${ }^{24,25}$

Stress has well-recognized deleterious health effects on diseases such as hypertension, as well as other cardiovascular and metabolic diseases. ${ }^{12,33-35}$ Stress was reported by the majority of our sample $(67.6 \%)$. The inclusion of select stress resiliency factors such as sleep, recovery, and social support is a unique aspect of this study that allowed a more comprehensive and relevant evaluation of the stress-health relation. Congruent with the relevant literature, sleep and recovery were inversely related to stress. ${ }^{36-38}$

In the present study, white respondents reported more stress than their African-American counterparts. This finding comes at odds with most of the reported literature, which shows higher stress among African Americans compared with whites because of racism-related stress and challenging socioeconomic experiences. ${ }^{39-41}$ However, the integration of several ethnic, racial, and religious minorities in metropolitan Detroit, where minority groups are no longer the exception but rather the true fabric of the society, may provide a deeper sense of belonging and therefore may partly explain this discrepancy. Moreover, even though we modeled important stress resiliency factors including sleep, recovery, and social support, other important factors, such as individual coping styles and participation in religious and voluntary organizations, were not measured. ${ }^{42,43}$

Fatigue played an important role in the relationship between stress and perceived health. It fully mediated this relationship, which could mean that stress may lead to poor health outcomes when it is significant enough to drain stress resiliency resources and manifest as fatigue. When stress is not related to fatigue there is no impact of stress on perceived health. Fatigue has been previously conceptualized as a warning sign, indicating harmful accumulation of stress. ${ }^{44}$ Therefore, individual and contextual stressors should be assessed in patients presenting with fatigue where no clear explanation is ascertained. Appropriate individual- and aggregate-level stress management and resiliency enhancement approaches should be offered to avert the deterioration of perceived health.

Sleep predicted perceived health directly and indirectly through fatigue and stress. This agrees with the evidence linking sleep to stress, ${ }^{37,38,45}$ fatigue, ${ }^{36,46}$ and perceived health. ${ }^{47,48}$ Therefore, improving sleep may lead to better perceived health independent of stress and fatigue, possibly through inflammatory ${ }^{49}$ and neurobiological ${ }^{50}$ pathways.

The benefits of social support are well established ${ }^{51-54}$ and are highlighted in this study. In our sample, strong social support was predictive of positive health perceptions directly and indirectly through favorable effects on sleep, recovery, and fatigue. Interestingly, marital status and employment were not predictive of perceived health. Therefore, support garnered from social sources outside work and committed relationships has strong influence on health.

Perceived health could be easily ascertained by primary care providers by asking patients to rate their health: "How do you think your general 
health is now?" and "How do you think your general health will be next year?" These simple questions can help identify patients who are a higher risk of future morbidity and mortality. ${ }^{17,18,55}$ The inverse relationship between perceived health and the extent of general symptoms and diseases is plausible and supported by the literature. ${ }^{17,18,56,57}$ However, the cross-sectional nature of this study limited our ability to test the causal associations. The other limitations of the study are the reliance on self-reports of diseases, height, weight, and employment and the fact that it is not population based. In addition, although depression was not independently ascertained, this should not compromise the results because even though depression and fatigue overlap, depression represents a more severe form and is probably present in patients with extreme fatigue scores. Furthermore, the fatigue scale was constructed similar to a validated scale that can differentiate between fatigue and depression. $^{24}$

Nonetheless, this study has important strengths. It specifically targets a demographically and socioeconomically diverse primary care population. It is the first study, to our knowledge, that examines the causal role of fatigue between stress and health. The primary care focus of this study is an advantage because fatigue and stress are prevalent in primary care settings, where they often present in concert with other common and costly medically unexplained symptoms. ${ }^{6}$ The scales used in this study are short and simple and could be easily incorporated into clinical encounters to screen patients for stress and fatigue or to monitor their response to treatment. Perceived health was an appropriate outcome measure for its simplicity, subjectivity, and proven validity and clinical utility.

\section{Conclusion}

We were able in the present cross-sectional study of primary care patients to demonstrate the important causal role of fatigue between stress and perceived health and the favorable effects exhibited by sleep, recovery, and social support. Therefore, stress, when severe enough to deplete the recovery resources and cause fatigue, may lead to poor perceived health. Fatigue can signal unresolved stress and serve as a window of opportunity to intervene and avert the deterioration of health perceptions.
We acknowledge Mr. Joseph Monsur, BS, for distributing and collecting the surveys, and Ms. Candis Harris for data entry.

\section{References}

1. Ricci JA, Chee E, Lorandeau AL, Berger J. Fatigue in the US workforce: prevalence and implications for lost productive work time. J Occup Environ Med 2007;49:1-10.

2. Bombardier $\mathrm{CH}$, Buchwald D. Chronic fatigue, chronic fatigue syndrome, and fibromyalgia. Disability and health-care use. Med Care 1996;34:924-30.

3. Kahol K, Leyba M, Deka M, et al. Effect of fatigue on psychomotor and cognitive skills. Am J Surg 2008;195:195-204.

4. Bates DW, Schmitt W, Buchwald D, et al. Prevalence of fatigue and chronic fatigue syndrome in a primary care practice. Arch Intern Med 1993;153: 2759-65.

5. Cullen W, Kearney Y, Bury G. Prevalence of fatigue in general practice. Ir J Med Sci 2002;171:10-2.

6. Kirmayer LJ, Groleau D, Looper KJ, Dao MD. Explaining medically unexplained symptoms. Can J Psychiatry 2004;49:663-72.

7. Taylor RR, Jason LA. Sexual abuse, physical abuse, chronic fatigue, and chronic fatigue syndrome: a community-based study. J Nerv Ment Dis 2001;189: 709-15.

8. Van Houdenhove B, Egle UT. Fibromyalgia: a stress disorder? Piecing the biopsychosocial puzzle together. Psychother Psychosom 2004;73:267-75.

9. Ridsdale L, Evans A, Jerrett W, Mandalia S, Osler K, Vora H. Patients who consult with tiredness: frequency of consultation, perceived causes of tiredness and its association with psychological distress. Br J Gen Pract 1994;44:413-6.

10. Thorsteinsson EB, Brown RF. Mediators and moderators of the stressor-fatigue relationship in nonclinical samples. J Psychosom Res 2009;66:21-9.

11. Weik U, Herforth A, Kolb-Bachofen V, Deinzer R. Acute stress induces proinflammatory signaling at chronic inflammation sites. Psychosom Med 2008; 70:906-12.

12. Branth S, Ronquist G, Stridsberg M, et al. Development of abdominal fat and incipient metabolic syndrome in young healthy men exposed to long-term stress. Nutr Metab Cardiovasc Dis 2007;17:427-35.

13. Atherton K, Wiles NJ, Lecky FE, et al. Predictors of persistent neck pain after whiplash injury. Emerg Med J 2006;23:195-201.

14. Fortin M, Bravo G, Hudon C, Lapointe L, Dubois MF, Almirall J. Psychological distress and multimorbidity in primary care. Ann Fam Med 2006;4:41722.

15. Hamer M, Chida Y, Molloy GJ. Psychological distress and cancer mortality. J Psychosom Res 2009; 66:255-8.

16. Halford C, Anderzen I, Arnetz B. Endocrine mea- 
sures of stress and self-rated health: a longitudinal study. J Psychosom Res 2003;55:317-20.

17. Kaplan GA, Goldberg DE, Everson SA, et al. Perceived health status and morbidity and mortality: evidence from the Kuopio ischemic heart disease risk factor study. Int J Epidemiol 1996;25:259-65.

18. Idler EL, Angel RJ. Self-rated health and mortality in the NHANES-I Epidemiologic Follow-up Study. Am J Public Health 1990;80:446-52.

19. Farmer MM, Ferraro KF. Distress and perceived health: mechanisms of health decline. J Health Soc Behav 1997;38:298-311.

20. Rohrer JE, Arif A, Denison A, Young R, Adamson S. Overall self-rated health as an outcome indicator in primary care. J Eval Clin Pract 2007;13:882-8.

21. Rohrer JE, Arif A, Denison A, Young R, Adamson S. Overall self-rated health: a new quality indicator for primary care. J Eval Clin Pract 2007;13:150-3.

22. Young RF, Schwartz KL, Monsur JC, West P, Neale AV. Primary care of overweight children: the importance of parent weight and attitudes about overweight: a MetroNet study. J Am Board Fam Med 2008;21:361-3.

23. Andersson C, Johnsson KO, Berglund M, Ojehagen A. Measurement properties of the Arnetz and Hasson stress questionnaire in Swedish university freshmen. Scand J Public Health 2009;37:273-9.

24. Arnetz BB, Frenzel L, Åkerstedt T, Lisspers J. The Brief Fatigue Syndrome Scale: validation and utilization in fatigue recovery studies. In: Watanabe Y, Evengard B, Natelson BH, Jason LA, Kuratsune H, eds. Fatigue science for human health. New York: Springer; 2008:55-66.

25. Anderzen I, Arnetz BB. The impact of a prospective survey-based workplace intervention program on employee health, biologic stress markers, and organizational productivity. J Occup Environ Med 2005; 47:671-82.

26. Boksem MA, Tops, M. Mental fatigue: costs and benefits. Brain Res Rev 2008;59:125-39.

27. Hu LB, Bentker PM, Cutoff criteria for fit indexes in covariance structure analysis: conventional criteria versus new alternatives. Struct Equ Modeling 1999; 6:1-55.

28. Skapinakis P, Lewis G, and Meltzer H, Clarifying the relationship between unexplained chronic fatigue and psychiatric morbidity: results from a community survey in Great Britain. Int Rev Psychiatry 2003;15: 57-64.

29. David A, Pelosi A, McDonald D, et al. Tired, weak, or in need of rest: fatigue among general practice attenders. BMJ 1990;301:1199-202.

30. Furberg $H$, Olarte $M$, Afari $N$, et al. The prevalence of self-reported chronic fatigue in a US twin registry. J Psychosom Res 2005;59:283-90.

31. Skapinakis P, Lewis G, and Mavreas V. Cross-cultural differences in the epidemiology of unexplained fatigue syndromes in primary care. Br J Psychiatry 2003;182:205-9.

32. Sharpe M, Wilks D. ABC of psychological medicine: fatigue. BMJ 2002;325:480-3.

33. Dimsdale JE. Psychological stress and cardiovascular disease. J Am Coll Cardiol 2008;51:1237-46.

34. Strike PC, Steptoe A. Behavioral and emotional triggers of acute coronary syndromes: a systematic review and critique. Psychosom Med 2005;67:179-86.

35. Schneider RH, Alexander CN, Staggers F, et al. Long-term effects of stress reduction on mortality in persons $\geq 55$ years of age with systemic hypertension. Am J Cardiol 2005;295:1060-4.

36. Hamilton NA, Affleck G, Tennen H, et al. Fibromyalgia: the role of sleep in affect and in negative event reactivity and recovery. Health Psychol 2008; 27:490-7.

37. Edell-Gustafsson UM, Kritz EI, Bogren IK. Selfreported sleep quality, strain and health in relation to perceived working conditions in females. Scand J Caring Sci 2002;16:179-87.

38. Killgore WDS, Kahn-Greene E, Lipissi E, Newman R, Kamimori G, Balkin TJ. Sleep deprivation reduces perceived emotional intelligence and constructive thinking skills. Sleep Med 2008;9:517-26.

39. Giscombe CL, Lobel M. Explaining disproportionately high rates of adverse birth outcomes among African Americans: the impact of stress, racism, and related factors in pregnancy. Psychol Bull 2005;131: 662-83.

40. Woods-Giscombe CL, Lobel, M. Race and gender matter: a multidimensional approach to conceptualizing and measuring stress in African American women. Cultur Divers Ethnic Minor Psychol 2008; 14:173-82.

41. Troxel WM, Mathews KA, Bromberger JT, SuttonTyrrell K. Chronic stress burden, discrimination, and subclinical carotid artery disease in African American and Caucasian women. Health Psychol 2003;2022:300-9.

42. Chatters LM, Bullard KM, Taylor RJ, Woodward AT, Neighbors HW, Jackson JS. Religious participation and DSM-IV disorders among older African Americans: findings from the National Survey of American Life. Am J Geriatr Psychiatry 2008;16: 957-65.

43. Veenstra G, Luginaah I, Wakefield S, Birch S, Eyles J, Elliot S. Who you know, where you live: social capital, neighborhood and health. Soc Sci Med 2005; 60:2799-818.

44. Watanabe Y. Preface and mini-review: fatigue science for human health. In: Watanabe Y, Evengard B, Natelson BH, Jason LA, Kuratsune H, eds. Fatigue science for human health. New York: Springer; 2008:v-xi.

45. Novati A, Roman V, Cetin T, et al. Chronically restricted sleep leads to depression-like changes in neurotransmitter receptor sensitivity and neuroen- 
docrine stress reactivity in rats. Sleep 2008;31:157985.

46. Pagani M, Pizzinelli P, Traon AP, et al, Hemodynamic, autonomic and baroreflex changes after one night sleep deprivation in healthy volunteers. Auton Neurosci 2009;145:76-80.

47. Mahon NE. The contributions of sleep to perceived health status during adolescence. Public Health Nurs 1995;12:127-33.

48. Unden AL, Elofsson S. Do different factors explain self-rated health in men and women? Gend Med 2006;3:295-308.

49. Simpson N, Dinges DF. Sleep and inflammation. Nutr Rev 2007;65(12 Pt 2):S244-52.

50. Chen JR, Wang TJ, Huang HY, et al. Fatigue reversibly reduced cortical and hippocampal dendritic spines concurrent with compromise of motor endurance and spatial memory. Neuroscience 2009;161: 1104-13.

51. Cobb S. Presidential address, 1976. Social support as a moderator of life stress. Psychosom Med 1976;38: $300-14$.
52. Matthew KA, Raikkonen K, Gallo L, Kuller LH. Association between socioeconomic status and metabolic syndrome in women: testing the reserve capacity model. Health Psychol 2008;27:576-83.

53. Cohen S, Wills TA. Stress, social support, and the buffering hypothesis. Psychol Bull 1985;98:310-57.

54. Unden AL, Orth-Gomer K, Elofsson S. Cardiovascular effects of social support in the work place: twenty-four-hour ECG monitoring of men and women. Psychosom Med 1991;53:50-60.

55. Farkas J, Nabb S, Zaletel-Kragelj L, Cleland JG, Lainscak M. Self-rated health and mortality in patients with chronic heart failure. Eur J Heart Fail 2009;11:518-24.

56. Van Der Windt DA, Dun KM, Spines-Dorgelo MN, Mallen CD, Blankenstein AH, Stalman WA. Impact of physical symptoms on perceived health in the community. J Psychosom Res 2008;64:265-74.

57. Rohrer JE, Bernard ME, Zhang Y, Rasmussen NH, Woroncow H. Marital status, feeling depressed and self-rated health in rural female primary care patients. J Eval Clin Pract 2008;14:214-7. 